\title{
Tamanho de amostra para a estimação da média de caracteres morfológicos e produtivos de nabo forrageiro
}

\author{
Sample size to estimate the average of morphological and productive traits of turnip
}

\author{
Alberto Cargnelutti Filho ${ }^{\mathrm{I}^{*}}$ Giovani Facco ${ }^{\mathrm{II}}$ Alessandro Dal'Col Lúcio ${ }^{\mathrm{I}}$ Marcos Toebe $^{\mathrm{II}}$ \\ Cláudia Burin ${ }^{\mathrm{II}}$ André Luis Fick ${ }^{\mathrm{III}}$ Ismael Mario Márcio Neu ${ }^{\mathrm{IV}}$
}

\begin{abstract}
O objetivo deste trabalho foi determinar o tamanho de amostra (número de plantas) para a estimação da média de caracteres morfológicos e produtivos de nabo forrageiro (Raphanus sativus L.). Em um experimento a campo, foram selecionadas, aleatoriamente, 300 plantas e mensurados os caracteres morfológicos (estatura de planta e número de folhas aos 21, 35, 48, 55, 62, 70, 77 e 91 dias após a semeadura) e os produtivos (massas verde e seca, de raízes, de caule, de folhas $e$ total aos 91 dias após a semeadura). Foram calculadas medidas de tendência central e de variabilidade, verificada a normalidade e calculado o tamanho de amostra. Para estimar a média, com mesma precisão, o tamanho de amostra dos caracteres produtivos é maior que dos morfológicos. Para os caracteres morfológicos e produtivos estudados, 231 plantas são suficientes para um erro de estimação máximo de $10 \%$ da média estimada, com grau de confiança de $95 \%$.
\end{abstract}

Palavras-chave: Raphanus sativus L., planejamento experimental, amostragem.

\section{ABSTRACT}

The aim of this research was to determine the sample size to estimate the average of morphological and productive traits of turnip (Raphanus sativus L.). In a experiment, 300 plants were randomly selected and measured for morphological traits (plant height and number of leaf at 21, 35, 48, 55, 62, 70, 77 and 91 days after sowing) and productive (fresh and dry matter of roots, stem, leaf and total of 91 days after sowing). Measures of central tendency and variability were calculated, normality was checked and the sample size was calculated. The sample size of productive traits is greater than morphological to estimate average and it has the same precision. For the morphological and productive traits studied 231 plants are enough to predict the average, with an estimation error maximum of $10 \%$ of estimated average, with a degree confidence of $95 \%$.

Key words: Raphanus sativus L., experimental planning, sampling.

O nabo forrageiro (Raphanus sativus L.) é uma planta anual, pertencente à família Brassicacea, herbácea, ereta e ramificada, podendo atingir até 180 $\mathrm{cm}$ de altura (DERPSCH \& CALEGARI, 1992). É cultivado em sistemas de rotação de culturas, devido ao rápido crescimento e cobertura do solo. No florescimento, apresenta produção média de massa seca da parte aérea de $2.938 \mathrm{~kg} \mathrm{ha}^{-1}$ (CRUSCIOL et al., 2005), 3.000kg ha-1 (DERPSCH \& CALEGARI, 1992) e $5.480,5 \mathrm{~kg} \mathrm{ha}^{-1}$ (LIMA et al., 2007).

$\mathrm{Na}$ condução de experimentos agrícolas, fatores como o tempo, a mão-de-obra e os recursos financeiros, se limitantes, dificultam a mensuração de muitos caracteres em muitas plantas. Assim, a mensuração desses caracteres por meio de uma amostra é um procedimento utilizado nesses experimentos. É comum, entre os pesquisadores, o questionamento sobre o tamanho de amostra adequado para que a amostra seja representativa da população de plantas. O tamanho de amostra é diretamente proporcional à variabilidade dos dados e ao grau de confiança desejado na estimativa e inversamente proporcional

IDepartamento de Fitotecnia, Centro de Ciências Rurais (CCR), Universidade Federal de Santa Maria (UFSM), 97105-900, Santa Maria,

RS, Brasil. E-mail: alberto.cargnelutti.filho@gmail.com.*Autor para correspondência.

IIPrograma de Pós-graduação em Agronomia, UFSM, Santa Maria, RS, Brasil.

IIICurso de Engenharia Florestal, UFSM, Santa Maria, RS, Brasil.

${ }^{\text {IV }}$ Curso de Agronomia, UFSM, Santa Maria, RS, Brasil. 
ao erro de estimação permitido, fixado a priori pelo pesquisador (BUSSAB \& MORETTIN, 2004).

Estudos sobre o dimensionamento do tamanho ótimo de parcela para avaliar a massa verde de nabo forrageiro (CARGNELUTTI FILHO et al., 2011) e do tamanho de amostra para a estimação da média de caracteres de crambe (CARGNELUTTI FILHO et al., 2010) têm sido realizados e mostraram resultados importantes para o planejamento experimental dessas culturas. No entanto, estudos sobre o dimensionamento do tamanho de amostra para a estimação da média de caracteres de nabo forrageiro não foram encontrados na literatura. Assim, o objetivo deste trabalho foi determinar o tamanho de amostra para a estimação da média de caracteres morfológicos e produtivos de nabo forrageiro.

Foi conduzido um experimento com nabo forrageiro em uma área experimental de $15 \mathrm{~m} \times 15 \mathrm{~m}$ $\left(225 \mathrm{~m}^{2}\right)$. A área experimental foi composta de 75 fileiras de $15 \mathrm{~m}$ de comprimento, espaçadas de $0,20 \mathrm{~m}$. A semeadura foi realizada em 10/06/2010 e a densidade foi ajustada para 15 plantas por metro linear. A adubação de base foi de $30 \mathrm{~kg} \mathrm{ha}^{-1}$ de $\mathrm{N}$, $150 \mathrm{~kg} \mathrm{ha}^{-1}$ de $\mathrm{P}_{2} \mathrm{O}_{5}$ e $150 \mathrm{~kg} \mathrm{ha}^{-1}$ de $\mathrm{K}_{2} \mathrm{O}$. Aos 28 dias após a emergência, foram aplicados $100 \mathrm{~kg}$ de $\mathrm{N} \mathrm{ha}^{-1}$. Foram selecionadas, aleatoriamente, 300 plantas e mensurados 16 caracteres morfológicos (estatura de planta, em cm, e número de folhas aos 21, 35, 48, $55,62,70,77$ e 91 dias após a semeadura) e oito produtivos (massas verde e seca, em gramas planta ${ }^{-1}$, de raízes, de caule, de folhas e total aos 91 dias após a semeadura, ou seja, no florescimento).

Para cada um dos 24 caracteres, foram calculadas as estatísticas: mínimo, máximo, média, mediana, variância, desvio padrão, erro padrão da média e coeficiente de variação. A seguir, foi verificada a normalidade dos dados, por meio do teste de Kolmogorov-Smirnov. Para cada caractere, tomando-se por base as 300 plantas, foi calculado o tamanho de amostra $(\eta)$ para as semiamplitudes do intervalo de confiança (erro de estimação) iguais a 2, $4,6, \ldots, 20 \%$ da estimativa da média, com grau de confiança (1- $\alpha)$ de $95 \%$, por meio da expressão

$$
\left.\eta=\frac{\mathrm{t}_{\alpha / 2}^{2} \mathrm{~s}^{2}}{(\text { erro de estimação) }}\right)^{2} \text { (BUSSAB \& MORETTIN, }
$$

2004), na qual $t_{\alpha / 2}$ é o valor crítico da distribuição $t$ de Student, cuja área à direita é igual a $\alpha / 2$, isto é, o valor de $\mathrm{t}$ tal que $\mathrm{P}\left(\mathrm{t}>\mathrm{t}_{\alpha / 2}\right)=\alpha / 2$, com $(\mathrm{n}-1)$ graus de liberdade, com $\alpha=5 \%$ de probabilidade de erro, e $\mathrm{s}^{2}$ é a estimativa de variância. A seguir, fixou-se $\eta$ em 300 plantas e foi calculado o erro de estimação em percentagem da estimativa da média (m), por meio da expressão, erro de estimação $=100 \frac{\mathrm{t}_{\alpha / 2} \mathrm{~s}}{\sqrt{\eta} \mathrm{m}}$ em que s é a estimativa do desvio padrão.

A média de massa seca da parte aérea (massa seca de caule + folhas) de nabo forrageiro foi 4,11 gramas planta $^{-1}$, equivalente a $3.082,5 \mathrm{~kg}$ ha $^{-1}$ (Tabela 1). Resultados semelhantes foram obtidos por DERPSCH \& CALEGARI (1992), CRUSCIOL et al. (2005) e LIMA et al. (2007), o que revela desenvolvimento adequado das plantas desse experimento.

Os baixos valores-p do teste de normalidade de Kolmogorov-Smirnov $(\mathrm{P} \leq 0,021)$, em relação aos caracteres morfológicos e produtivos das 300 plantas, com exceção da estatura de planta avaliada aos 91 dias após a semeadura $(\mathrm{P}=0,226)$, revelaram não aderência dos dados à distribuição normal (Tabela 1). Embora a população básica seja não normal, conforme o teorema limite central, a distribuição da média amostral será aproximadamente normal para amostras superiores a 30 observações (BUSSAB \& MORETTIN, 2004). Então, diante dessas considerações, pode-se inferir que esses dados são adequados ao estudo do dimensionamento de amostra, com base na distribuição t de Student. O tamanho de amostra, nesse caso, também poderia ser estimado, com base na distribuição normal. No entanto, por gerar resultados similares aos obtidos com base na distribuição normal e o estudo ser com base em amostragem (300 plantas), a opção foi dimensionar a partir da distribuição t de Student.

A magnitude do coeficiente de variação (CV) oscilou entre 14,95\% para o número de folhas aos 70 dias após a semeadura (NF70) e 77,07\% para a massa verde de raízes aos 91 dias após a semeadura (MVR) e a média dos 24 caracteres foi 44,46\% (Tabela 1). De maneira geral, o CV dos caracteres produtivos (média dos oito caracteres $=68,96 \%$ ) foi, aproximadamente, 2,1 vezes maior que o $\mathrm{CV}$ dos caracteres morfológicos (média dos 16 caracteres $=32,20 \%$ ). A maior variabilidade dos caracteres produtivos em relação aos morfológicos, pode, provavelemente, ser devida à maior intereferência do ambiente sobre os caracteres produtivos avaliados somente no final do experimento (neste estudo, aos 91 dias após a semeadura). Em caracteres de crambe, CARGNELUTTI FILHO et al. (2010) verificaram relação semelhante a essa, ou seja, o CV dos caracteres produtivos (média de três caracteres $=56,77 \%$ ) foi, aproximadamente, 2,4 vezes maior que o $\mathrm{CV}$ dos caracteres morfológicos (média de três caracteres $=23,78 \%$ ). Isso sugere que, para a 
Tabela 1 - Mínimo (min), máximo (max), média (m), mediana (med), variância $\left(\mathrm{s}^{2}\right)$, desvio padrão (s), erro padrão da média (ep), coeficiente de variação $(\mathrm{CV})$ e valor-p do teste de Kolmogorov-Smirnov de caracteres morfológicos e produtivos de nabo forrageiro (Raphanus sativus L.) avaliados em 300 plantas.

\begin{tabular}{|c|c|c|c|c|c|c|c|c|c|}
\hline Caractere $^{(1)}$ & $\min$ & $\max$ & $\mathrm{m}$ & med & $s^{2}$ & s & ep & $\mathrm{CV}(\%)$ & valor-p \\
\hline & \multicolumn{9}{|c|}{ 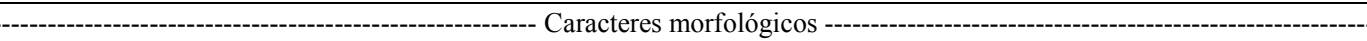 } \\
\hline EP21 & 1,10 & 3,30 & 1,82 & 1,70 & 0,18 & 0,42 & 0,02 & 23,35 & 0,001 \\
\hline EP35 & 1,10 & 3,30 & 1,87 & 1,80 & 0,19 & 0,43 & 0,03 & 23,20 & 0,000 \\
\hline EP48 & 1,10 & 7,00 & 2,22 & 2,00 & 0,77 & 0,88 & 0,05 & 39,36 & 0,000 \\
\hline EP55 & 1,10 & 13,40 & 3,15 & 2,50 & 3,84 & 1,96 & 0,11 & 62,19 & 0,000 \\
\hline EP62 & 1,20 & 29,20 & 6,80 & 5,00 & 25,72 & 5,07 & 0,29 & 74,61 & 0,000 \\
\hline EP70 & 1,80 & 53,50 & 21,08 & 18,95 & 143,20 & 11,97 & 0,69 & 56,77 & 0,019 \\
\hline EP77 & 9,00 & 89,00 & 54,98 & 57,50 & 268,43 & 16,38 & 0,95 & 29,80 & 0,021 \\
\hline EP91 & 29,00 & 132,20 & 86,40 & 88,15 & 256,43 & 16,01 & 0,92 & 18,53 & 0,226 \\
\hline NF21 & 0,00 & 2,00 & 1,43 & 2,00 & 0,51 & 0,71 & 0,04 & 49,78 & 0,000 \\
\hline NF35 & 1,00 & 5,00 & 3,38 & 3,00 & 0,68 & 0,83 & 0,05 & 24,49 & 0,000 \\
\hline NF48 & 2,00 & 8,00 & 4,75 & 5,00 & 0,80 & 0,89 & 0,05 & 18,83 & 0,000 \\
\hline NF55 & 3,00 & 9,00 & 5,56 & 5,00 & 0,85 & 0,92 & 0,05 & 16,56 & 0,000 \\
\hline NF62 & 4,00 & 11,00 & 6,15 & 6,00 & 1,17 & 1,08 & 0,06 & 17,59 & 0,000 \\
\hline NF70 & 4,00 & 10,00 & 6,89 & 7,00 & 1,06 & 1,03 & 0,06 & 14,95 & 0,000 \\
\hline NF77 & 4,00 & 11,00 & 6,64 & 7,00 & 1,52 & 1,23 & 0,07 & 18,58 & 0,000 \\
\hline NF91 & 2,00 & 14,00 & 7,30 & 7,00 & 3,78 & 1,94 & 0,11 & 26,64 & 0,000 \\
\hline MVR & 0,41 & 18,83 & 4,19 & ------ & $\begin{array}{c}\text { res produ } \\
10,45\end{array}$ & s ----- & 0,19 & 77,07 & 0,000 \\
\hline MVC & 3,10 & 126,66 & 38,80 & 34,13 & 587,30 & 24,23 & 1,40 & 62,47 & 0,013 \\
\hline MVF & 0,46 & 51,16 & 13,26 & 10,96 & 84,53 & 9,19 & 0,53 & 69,33 & 0,002 \\
\hline MVT & 4,54 & 180,57 & 56,25 & 50,21 & 1236,45 & 35,16 & 2,03 & 62,51 & 0,004 \\
\hline MSR & 0,07 & 2,17 & 0,55 & 0,45 & 0,14 & 0,38 & 0,02 & 69,18 & 0,000 \\
\hline MSC & 0,19 & 13,71 & 3,70 & 2,99 & 6,46 & 2,54 & 0,15 & 68,59 & 0,000 \\
\hline MSF & 0,03 & 5,19 & 1,46 & 1,12 & 1,18 & 1,09 & 0,06 & 74,76 & 0,000 \\
\hline MST & 0,38 & 20,64 & 5,71 & 4,63 & 14,97 & 3,87 & 0,22 & 67,79 & 0,000 \\
\hline
\end{tabular}

(1) EP21, EP35, EP48, EP55, EP62, EP70, EP77 e EP91: estatura de planta, em cm, aos 21, 35, 48, 55, 62, 70, 77 e 91 dias após a semeadura, respectivamente; NF21, NF35, NF48, NF55, NF62, NF70, NF77 e NF91: número de folhas aos 21, 35, 48, 55, 62, 70, 77 e 91 dias após a semeadura, respectivamente; MVR, MVC, MVF e MVT: massa verde, em g, de raízes, caule, folhas e total, respectivamente, aos 91 dias após a semeadura; MSR, MSC, MSF e MST: massa seca, em g, de raízes, de caule, de folhas e total, respectivamente, aos 91 dias após a semeadura.

obtenção da estimativa da média, com determinada precisão, o tamanho de amostra de caracteres produtivos é maior em relação aos morfológicos. Embora o experimento não tenha sido repetido em mais épocas ou anos, é provável que, pelo número expressivo de plantas avaliadas (300), a variabilidade tenha sido contemplada adequadamente. Além disso, possíveis outliers não foram eliminados, pois a variabilidade existente é importante e confere adequabilidade ao estudo do dimensionamento de amostra de cada caractere. A retirada de outliers poderia subestimar o tamanho de amostra.

O tamanho de amostra, para a estimação da média de cada caractere, com semiamplitude do intervalo de confiança igual a $2 \%$ da estimativa da média (m) e grau de confiança de $95 \%$, oscilou entre 217 plantas para o número de folhas aos 70 dias após a semeadura (NF70) e 5.751 plantas para a massa verde de raízes aos 91 dias após a semeadura (MVR). Esses resultados confirmam a variabilidade existente entre os caracteres, assim como verificado em crambe (CARGNELUTTI FILHO et al., 2010).

Para alguns caracteres de nabo forrageiro, aqueles com menor variabilidade, é possível estimar a média com erro de estimação de $2 \%$. Por outro lado, para os caracteres com maior variabilidade é difícil obter estimativas da média com essa mesma precisão, em função do elevado número de plantas a serem mensuradas. Nesse caso, a opção por menores tamanhos de amostra proporcionariam maiores erros de estimação (menor precisão). Assim, menores tamanhos de amostra foram estimados com base em semiamplitudes do intervalo de confiança iguais a 4, $6, \ldots, 20 \%$ da média (Tabela 2). Na cultura de nabo forrageiro, essas combinações de erro de estimação e tamanho de amostra, juntamente com o tamanho 
Tabela 2 - Tamanho de amostra (número de plantas) para a estimação da média de caracteres morfológicos e produtivos de nabo forrageiro (Raphanus sativus L.), para as semiamplitudes do intervalo com 95\% de confiança, iguais a 2, 4, 6, ..., 20\% da média e semiamplitude do intervalo de confiança em percentagem da estimativa da média do caractere (Erro \%), com base em 300 plantas.

\begin{tabular}{|c|c|c|c|c|c|c|c|c|c|c|c|}
\hline Caractere $^{(1)}$ & $2 \%$ & $4 \%$ & $6 \%$ & $8 \%$ & $10 \%$ & $12 \%$ & $14 \%$ & $16 \%$ & $18 \%$ & $20 \%$ & Erro\% \\
\hline & \multicolumn{11}{|c|}{ 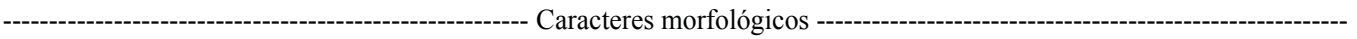 } \\
\hline EP21 & 528 & 132 & 59 & 33 & 22 & 15 & 11 & 9 & 7 & 6 & 2,65 \\
\hline EP35 & 522 & 131 & 58 & 33 & 21 & 15 & 11 & 9 & 7 & 6 & 2,64 \\
\hline EP48 & 1.501 & 376 & 167 & 94 & 61 & 42 & 31 & 24 & 19 & 16 & 4,47 \\
\hline EP55 & 3.745 & 937 & 417 & 235 & 150 & 105 & 77 & 59 & 47 & 38 & 7,07 \\
\hline EP62 & 5.390 & 1.348 & 599 & 337 & 216 & 150 & 110 & 85 & 67 & 54 & 8,48 \\
\hline EP70 & 3.121 & 781 & 347 & 196 & 125 & 87 & 64 & 49 & 39 & 32 & 6,45 \\
\hline EP77 & 860 & 215 & 96 & 54 & 35 & 24 & 18 & 14 & 11 & 9 & 3,39 \\
\hline EP91 & 333 & 84 & 37 & 21 & 14 & 10 & 7 & 6 & 5 & 4 & 2,11 \\
\hline NF21 & 2.400 & 600 & 267 & 150 & 96 & 67 & 49 & 38 & 30 & 24 & 5,66 \\
\hline NF35 & 581 & 146 & 65 & 37 & 24 & 17 & 12 & 10 & 8 & 6 & 2,78 \\
\hline NF48 & 344 & 86 & 39 & 22 & 14 & 10 & 8 & 6 & 5 & 4 & 2,14 \\
\hline NF55 & 266 & 67 & 30 & 17 & 11 & 8 & 6 & 5 & 4 & 3 & 1,88 \\
\hline NF62 & 300 & 75 & 34 & 19 & 12 & 9 & 7 & 5 & 4 & 3 & 2,00 \\
\hline NF70 & 217 & 55 & 25 & 14 & 9 & 7 & 5 & 4 & 3 & 3 & 1,70 \\
\hline NF77 & 335 & 84 & 38 & 21 & 14 & 10 & 7 & 6 & 5 & 4 & 2,11 \\
\hline NF91 & 688 & 172 & 77 & 43 & 28 & 20 & 15 & 11 & 9 & 7 & 3,03 \\
\hline MVR & 5.751 & 1.438 & 639 & 360 & $\begin{array}{c}--- \text { Car } \\
231\end{array}$ & $\begin{array}{c}\text { eres pro } \\
160\end{array}$ & $\begin{array}{r}\text { vos -- } \\
118\end{array}$ & 90 & 71 & 58 & 8,76 \\
\hline MVC & 3.779 & 945 & 420 & 237 & 152 & 105 & 78 & 60 & 47 & 38 & 7,10 \\
\hline MVF & 4.655 & 1.164 & 518 & 291 & 187 & 130 & 95 & 73 & 58 & 47 & 7,88 \\
\hline MVT & 3.784 & 946 & 421 & 237 & 152 & 106 & 78 & 60 & 47 & 38 & 7,10 \\
\hline MSR & 4.634 & 1.159 & 515 & 290 & 186 & 129 & 95 & 73 & 58 & 47 & 7,86 \\
\hline MSC & 4.555 & 1.139 & 507 & 285 & 183 & 127 & 93 & 72 & 57 & 46 & 7,79 \\
\hline MSF & 5.412 & 1.353 & 602 & 339 & 217 & 151 & 111 & 85 & 67 & 55 & 8,49 \\
\hline MST & 4.449 & 1.113 & 495 & 279 & 178 & 124 & 91 & 70 & 55 & 45 & 7,70 \\
\hline
\end{tabular}

EP21, EP35, EP48, EP55, EP62, EP70, EP77 e EP91: estatura de planta, em cm, aos 21, 35, 48, 55, 62, 70, 77 e 91 dias após a semeadura, respectivamente; NF21, NF35, NF48, NF55, NF62, NF70, NF77 e NF91: número de folhas aos 21, 35, 48, 55, 62, 70, 77 e 91 dias após a semeadura, respectivamente; MVR, MVC, MVF e MVT: massa verde, em g, de raízes, caule, folhas e total, respectivamente, aos 91 dias após a semeadura; MSR, MSC, MSF e MST: massa seca, em g, de raízes, de caule, de folhas e total, respectivamente, aos 91 dias após a semeadura.

ótimo de parcela (CARGNELUTTI FILHO et al., 2011), podem ser utilizadas por pesquisadores para o planejamento adequado de seus experimentos.

Caso a opção seja amostrar 300 plantas, o erro de estimação médio seria de $\pm 3,66$ e $\pm 7,84 \%$ da estimativa da média $(\mathrm{m})$, respectivamente, para os caracteres morfológicos e produtivos (Tabela 2). Portanto, os resultados evidenciam que o tamanho de amostra de caracteres produtivos é maior que o de morfológicos, com exceção das estaturas de planta aos 55 e 62 dias após a semeadura, que foram similares aos caracteres produtivos. Em crambe, CARGNELUTTI FILHO et al. (2010) também concluíram que o tamanho de amostra para a estimação da média de caracteres produtivos é superior aos morfológicos. Na prática, para a estimação da média dos caracteres morfológicos e produtivos, 231 plantas são suficientes, para um erro de estimação máximo de $10 \%$ da média, com grau de confiança de $95 \%$.

\section{AGRADECIMENTOS}

Ao Conselho Nacional de Desenvolvimento Científico e Tecnológico (CNPq), à Coordenação de Aperfeiçoamento de Pessoal de Nível Superior (CAPES) e à Fundação de Amparo à Pesquisa do Estado do Rio Grande do Sul (FAPERGS), pela concessão de bolsa aos autores. À FAPERGS pelo auxílio financeiro.

\section{REFERÊNCIAS}

BUSSAB, W.O.; MORETTIN, P.A. Estatística básica. 5.ed. São Paulo: Saraiva, 2004. 526p.

CARGNELUTTI FILHO, A. et al. Tamanhos de parcela e de ensaio de uniformidade em nabo forrageiro. Ciência Rural, v.41, 
p.1-9, 2011. Disponível em: <http://www.scielo.br/pdf/cr/v41n9/ a9911 cr5182.pdf $>$. Acesso em: 07 dez. 2011. doi: 10.1590/S010384782011005000119.

CARGNELUTTI FILHO, A. et al. Tamanho de amostra e relações lineares de caracteres morfológicos e produtivos de crambe. Ciência Rural, v.40, p.2262-2267, 2010. Disponível em: <http:// www.scielo.br/pdf/cr/v40n11/a774cr3308.pdf>. Acesso em: 07 dez. 2011. doi: 10.1590/S0103-84782010001100003.

CRUSCIOL, C.A.C. et al. Persistência de palhada e liberação de nutrientes do nabo forrageiro no plantio direto. Pesquisa
Agropecuária Brasileira, v.40, p.161-168, 2005. Disponível em: $<$ http://www.scielo.br/pdf/pab/v40n2/23823.pdf>. Acesso em: 07 dez. 2011. doi: 10.1590/S0100-204X2005000200009.

DERPSCH, R.; CALEGARI, A. Plantas para adubação verde de inverno. Londrina: Iapar, 1992. 80p. (Circular, 73).

LIMA, J.D. et al. Comportamento do nabo forrageiro (Raphanus sativus L.) e da nabiça (Raphanus raphanistrum L.) como adubo verde. Pesquisa Agropecuária Tropical, v.37, p.60-63, 2007. Disponível em: <http://www.revistas.ufg.br/index.php/pat/article/ download/1871/1778>. Acesso em: 07 dez. 2011. 